\title{
Fourier decomposition of payoff matrix for symmetric three-strategy games
}

\author{
György Szabó, ${ }^{1,2}$ Kinga S. Bodó, ${ }^{3}$ Benjamin Allen, ${ }^{4}$ and Martin A. Nowak ${ }^{4,5}$ \\ ${ }^{1}$ Institute of Technical Physics and Materials Science, Research Centre for Natural Sciences, Hungarian Academy of Sciences, \\ P.O. Box 49, H-1525 Budapest, Hungary \\ ${ }^{2}$ Regional Knowledge Centre, Eötvös University, Irányi Dániel u. 4, H-8000 Székesfehérvár, Hungary \\ ${ }^{3}$ Roland Eötvös University, Institute of Physics, Pázmány P. sétány 1/A, H-1117 Budapest, Hungary \\ ${ }^{4}$ Program for Evolutionary Dynamics, Harvard University, One Brattle Square, Cambridge, Massachusetts 02138, USA \\ ${ }^{5}$ Department of Mathematics, Department of Organismic and Evolutionary Biology, Harvard University, \\ Cambridge, Massachusetts 02138, USA \\ (Received 30 July 2014; published 20 October 2014)
}

\begin{abstract}
In spatial evolutionary games the payoff matrices are used to describe pair interactions among neighboring players located on a lattice. Now we introduce a way how the payoff matrices can be built up as a sum of payoff components reflecting basic symmetries. For the two-strategy games this decomposition reproduces interactions characteristic to the Ising model. For the three-strategy symmetric games the Fourier components can be classified into four types representing games with self-dependent and cross-dependent payoffs, variants of three-strategy coordinations, and the rock-scissors-paper (RSP) game. In the absence of the RSP component the game is a potential game. The resultant potential matrix has been evaluated. The general features of these systems are analyzed when the game is expressed by the linear combinations of these components.
\end{abstract}

DOI: 10.1103/PhysRevE.90.042811

PACS number(s): 89.65.-s, 89.75. Fb, 87.23.Kg

\section{INTRODUCTION}

In many evolutionary games the pair interactions among the players are defined by payoff matrices [1-4]. In these models the players are located on the sites of a lattice or graph and they play two-person games with all their neighbors (to accumulate income or fitness) when using one of their possible strategies or states permitted in the system [5,6]. For the simplest models both the players and games are equivalent and this fact implies the straightforward application of the methods developed within the statistical physics for the investigation of many-particle systems. The different approaches and approximative methods can be exploited particularly for the so-called potential games $[7,8]$ because these systems develop into the Boltzmann distribution for suitable dynamical rules $[9,10]$ that define how the players can change their own strategy during the evolutionary process. The intimate relationship between physical models and the evolutionary potential games implies the direct adoption of methods and results for the analysis of living systems studied in biology [11] and social sciences [12-14].

Up to now the exhaustive and systematic analysis of these systems are prevented by the huge freedom realized by the large number of parameters quantifying the interactions (games), dynamical rules, and connectivity structures. For the sake of simplicity now our investigations are restricted to lattice models by assuming that the players are distributed on the sites of a square lattice and their payoffs are collected from four symmetric two- or three-strategy games that they play with their nearest neighbors. Additionally we assume that the players use identical dynamical rule to modify their strategy with a myopic purpose of increasing their own income. More precisely, for the two-strategy systems, the dynamical rule is similar to those suggested by Glauber [15] for the investigation of the kinetic Ising model $[16,17]$.

The similarity between the Ising model and the mentioned two-strategy evolutionary games has motivated us to separate the payoff matrix into components that have proper symmetries and application in physical systems. This idea will be illustrated in Sec. III. The latter approach is extended in the next section where the $3 \times 3$ payoff matrix is decomposed into its two-dimensional Fourier components. The general features of the spatial models when the games or interactions are defined by one or two components will be discussed in Sec. V. Section VI illustrates different variants of coordination games that exhibit an order-disorder phase transition analogous to that found for the three-state Potts model [18,19]). First, however, we define the framework and formalism in the following section.

\section{EVOLUTIONARY GAMES ON LATTICE}

We consider systems with equivalent players who are residing on the sites $x$ of a square lattice consisting of $N$ sites. The translation invariance is ensured by periodic boundary conditions whereas the sites are labeled $x=1, \ldots, N$. Each player $x$ chooses one of the $Q$ pure strategies denoted by $Q$-dimensional unit vectors as

$$
\mathbf{s}_{x}=\left(\begin{array}{c}
1 \\
0 \\
\vdots \\
0
\end{array}\right),\left(\begin{array}{c}
0 \\
1 \\
\vdots \\
0
\end{array}\right), \ldots,\left(\begin{array}{l}
0 \\
\vdots \\
0 \\
1
\end{array}\right) .
$$

Additionally, the strategy of the player $x$ will be labeled by an integer $s_{x}=1, \ldots, Q$ and the set $\mathbf{S}=\left(s_{1}, \ldots, s_{N}\right)$ of the individual strategies defines the microscopic state (or strategy profile in the terminology of game theory) of the whole system. In the present model, the income of player $x$ comes from games she plays with her four neighbors; that is, the accumulated payoff $u_{x}\left(s_{x}\right)$ can be expressed as

$$
u_{x}\left(s_{x}\right)=\sum_{\delta_{x}} \mathbf{s}_{x} \cdot \mathbf{A} \mathbf{s}_{x+\delta_{x}}
$$


where the summation runs over the four nearest-neighbor sites $x+\delta_{x}$. The $A_{j k}(j, k=1, \ldots, Q)$ element of the payoff matrix A defines the income for player $x$ if she chooses her $j$ th strategy whereas the opponent selects the $k$ th pure strategy. For symmetric games the interacting players $x$ and $y$ receive payoffs $\mathbf{s}_{x} \cdot \mathbf{A} \mathbf{s}_{y}$ and $\mathbf{s}_{y} \cdot \mathbf{A} \mathbf{s}_{x}$, respectively.

An important class of games, known as potential games, plays a crucial role in the study of evolutionary games in general. Potential games are defined by the existence of a potential value assigned to each strategy profile $\left(\mathbf{s}_{x}, \mathbf{s}_{y}\right)$. This potential value has the property that, if one player unilaterally changes strategy, the resulting change in potential equals the change in that players payoff [7]. Thus the change in potential quantifies the incentive for each player to change strategy. For a symmetric two-person game (defined by $\mathbf{A}$ ) the potential, if it exists, can be described by a symmetric potential matrix $\mathbf{V}$ that satisfies the following condition:

$$
\mathbf{s}_{x} \cdot \mathbf{A} \mathbf{s}_{y}-\mathbf{s}_{x}^{\prime} \cdot \mathbf{A} \mathbf{s}_{y}=\mathbf{s}_{x} \cdot \mathbf{V} \mathbf{s}_{y}-\mathbf{s}_{x}^{\prime} \cdot \mathbf{V} \mathbf{s}_{y},
$$

for any pure strategies denoted by the vectors $\mathbf{s}_{x}, \mathbf{s}_{x}^{\prime}$, and $\mathbf{s}_{y}$. The existence of potential involves that the sum of payoff variations [given by Eq. (3)] is zero along any closed loops within the space of strategy profiles and $\mathbf{V}$ is unique up to the addition of a constant. Furthermore, if the payoff matrix is symmetric; that is, $\mathbf{A}=\mathbf{A}^{+}\left(\mathbf{A}^{+}\right.$indicates the transpose of $\left.\mathbf{A}\right)$, then $\mathbf{V}=\mathbf{A}$. It is also known that, for the two-strategy symmetric matrix games the potential matrix exists and symmetric, i.e., $\mathbf{V}=\mathbf{V}^{+}$ $[7,20]$. Additionally, if a multi-player game is built up from two-player potential games then the potential for the whole system can be defined as a suitable sum of the pair potentials. Consequently, in the present lattice model the potential for any strategy profile $\mathbf{s}$ is given by the following expression:

$$
U(\mathbf{S})=\frac{1}{2} \sum_{x, \delta_{x}} \mathbf{s}_{x} \cdot \mathbf{V} \mathbf{s}_{x+\delta_{x}} ;
$$

that is, a scalar function of the strategy profile $\mathbf{s}$. If only a single player modifies her strategy from $s_{x}$ to $s_{x}^{\prime}$ then the variation of $U(\mathbf{s})$ is equivalent to the payoff variation of the given player $x$.

In the present systems we assume that, during the evolutionary process, randomly chosen players are allowed consecutively to choose another strategy in order to receive higher payoff. More precisely, within an elementary step of the evolutionary process player $x$ can modify her strategy to $s_{x}^{\prime}$ with a probability

$$
w\left(s_{x}^{\prime}\right)=\frac{e^{u_{x}\left(s_{x}^{\prime}\right) / K}}{\sum_{s_{x}} e^{u_{x}\left(s_{x}\right) / K}}
$$

favoring exponentially the strategy that provides the highest individual payoff. According to this so-called logit rule $[9,21-$ 23 ] the choice of the new strategy is independent of the initial state and it ensures a "local" Boltzmann distribution [24] in the individual strategy distribution for the given player at a quenched neighborhood. For this transition rule $K$ measures the magnitude of the noise or the uncertainties in the stochastic decision procedure. It is emphasized that for $Q=2$ this rule is equivalent to the probability of spin reversal suggested by Glauber [15] for the analysis of the kinetic Ising model where $K$ denotes the temperature.
Blume $[9,10]$ has shown that these systems evolve into the Boltzmann distribution where the probability of the microscopic state $\mathbf{s}$ is given as

$$
p(\mathbf{S})=\frac{1}{Z} e^{U(\mathbf{S}) / K}
$$

if the normalization is provided by

$$
Z=\sum_{\mathbf{s}} e^{U(\mathbf{S}) / K},
$$

where the summation runs over all possible microscopic states s. The latter feature implies the straightforward application of the results of statistical physics (including the problem of ergodicity for large systems at low noise levels [25]) when studying the macroscopic behavior of evolutionary games.

In order to explore the general features of these lattice systems for the given components we have performed Monte Carlo (MC) simulations. These MC simulations are used for displaying the spatial strategy arrangements for several types of games. For the quantitative analysis the strategy frequencies are determined when varying the noise level $K$. In most of the cases the relative statistical error is smaller than $1 \%$ due to the large system sizes ( $N$ is varied from $10^{5}$ to $2 \times 10^{6}$ ) and sufficiently long run times. In the close vicinity of the critical transitions the thermalization and sampling times have exceeded $10^{6}$ Monte Carlo step (MCS) (during 1 MCS each player has a chance once on average to modify her strategy).

\section{DECOMPOSITION OF 2 × 2 GAMES}

In this section we briefly illustrate the idea of decomposition that can be considered as a method for the anatomy of games. Accordingly, the payoff matrix of a symmetric $2 \times 2$ game can be written as

$$
\begin{aligned}
\mathbf{A}= & \left(\begin{array}{ll}
A_{11} & A_{12} \\
A_{21} & A_{22}
\end{array}\right)=A_{11}\left(\begin{array}{ll}
1 & 0 \\
0 & 0
\end{array}\right)+A_{12}\left(\begin{array}{ll}
0 & 1 \\
0 & 0
\end{array}\right) \\
& +A_{21}\left(\begin{array}{ll}
0 & 0 \\
1 & 0
\end{array}\right)+A_{22}\left(\begin{array}{ll}
0 & 0 \\
0 & 1
\end{array}\right),
\end{aligned}
$$

where the four matrices represent orthonormal basis vectors of a four-dimensional parameter space. Instead of the above natural basis "vectors" one can choose another set of the orthonormal basis vectors that reflects the inherent symmetries of the payoff matrices. For example, A may be written as

$$
\mathbf{A}=\sum_{n=1}^{4} \alpha(n) \mathbf{f}(n),
$$

where the matrices $\mathbf{f}(n)(n=1, \ldots, 4)$ define new orthonormal basis vectors that can be chosen as

$$
\begin{aligned}
& \mathbf{f}(1)=\frac{1}{2}\left(\begin{array}{ll}
1 & 1 \\
1 & 1
\end{array}\right), \quad \mathbf{f}(2)=\frac{1}{2}\left(\begin{array}{ll}
1 & -1 \\
1 & -1
\end{array}\right), \\
& \mathbf{f}(3)=\frac{1}{2}\left(\begin{array}{rr}
1 & 1 \\
-1 & -1
\end{array}\right), \quad \mathbf{f}(4)=\frac{1}{2}\left(\begin{array}{rr}
1 & -1 \\
-1 & 1
\end{array}\right) .
\end{aligned}
$$

The above matrices satisfy the conditions of orthogonality and normalization as

$$
\sum_{j, k=1}^{2} \mathbf{f}_{j k}(n) \mathbf{f}_{j k}(m)=\delta_{n m},
$$


where $n, m=1, \ldots, 4$ and $\delta_{n m}$ denotes the Kronecker's delta function $\left(\delta_{n m}=1\right.$ if $n=m$, otherwise $\left.\delta_{n m}=0\right)$. For this orthonormal set of the basis vectors the coefficients $\alpha(n)$ in Eq. (9) are given as

$$
\alpha(n)=\sum_{j, k=1}^{2} A_{j k} f_{j k}(n) .
$$

In this notation the first term corresponds to the average portion of payoffs. For most of the traditional and evolutionary games this constant term does not influence the individual choices. The first and second components together define the so-called cross-dependent part of payoffs that can only be influenced by the co-player(s). For such a type of payoff there is no direct interactions between the selfish and rational players for the noncooperative games. This type of interaction, however, becomes relevant for the so-called cooperative games when the players are allowed to collaborate with each other, as well as for evolutionary games when the strategy update is based on imitation [26]. The latter situations occur for games where $\alpha(3)=\alpha(4)=0$.

On the contrary, the third term $\mathbf{f}(3)$ together with $\mathbf{f}(1)$ define self-dependent payoffs that are independent of the other's choice. In that case both selfish players can easily maximize her own payoff in parallel. Notice that the direct interactions between the players are missing for logit type (myopic) strategy updates if $\alpha(4)=0$. The direct interaction between the players are described by $\mathbf{f}(4)$ which is equivalent to the coordination game. More precisely, $\mathbf{A}=\alpha(4) \mathbf{f}(4)$ is a coordination (anticoordination) game for $\alpha(4)>0[\alpha(4)<0]$.

For the terminology of magnetic Ising model [17,20] the fourth component $\mathbf{f}(4)$ describes the pair interaction between two equivalent neighboring spins that can be oriented "upward" or "downward." The corresponding coefficient $\alpha(4)$ defines the strength of interaction (coupling constant) favoring either ferromagnetic $[\alpha(4)>0]$ or antiferromagnetic $[\alpha(4)<0]$ spin arrangements. On the other hand, the effect of the external magnetic field is similar to the self-dependent components.

Due to the linear relationship between the potential and payoff matrices [see Eq. (3)] the potential matrix $\mathbf{V}$ can also be decomposed into a sum as

$$
\mathbf{V}=\sum_{n} \alpha(n) \mathbf{v}(n),
$$

where $\mathbf{v}(n)$ is the potential for the pair interaction defined by the payoff matrix $\mathbf{A}=\mathbf{f}(n)$, if it exists. For the evaluation of the corresponding potential components one can exploit the symmetries. Namely, for the cross-dependent components $\mathbf{v}(1)=\mathbf{v}(2)=0$ as the payoff of player $x$ is modified by herself when choosing another strategy. Additionally, one can easily check that, for the third component, the potential matrix can be given as $\mathbf{v}(3)=\mathbf{f}(3)+\mathbf{f}^{+}(3)$. Furthermore, $\mathbf{v}(4)=\mathbf{f}(4)$ because it is a symmetric matrix. Consequently, the potential matrix exist for all the symmetric $2 \times 2$ matrix games and the corresponding potential depends only on two components, namely,

$$
\mathbf{V}=\alpha(3)\left[\mathbf{f}(3)+\mathbf{f}^{+}(3)\right]+\alpha(4) \mathbf{f}(4)
$$

Evidently, there are many other possibilities for choosing an adequate set of basis vectors. The above choice is motivated by the intimate analogy between the symmetric $2 \times 2$ matrix games and Ising models. On the one hand, the large number of possibilities (particulary for $Q>2$ ) provides additional points of view for the anatomy of games. On the other hand, this freedom in the selection is accompanied with difficulties related to the absence of additional criteria supporting an optimal choice. In the next section we suggest a way that seems to be convenient for $Q=3$.

\section{FOURIER DECOMPOSITION OF 3 × 3 GAMES}

The Fourier decomposition of the $Q \times Q$ payoff matrix is based on the analogy when the payoffs $A_{j k}$ are considered as one-dimensional displacements of atoms at the sites $(j, k)(j, k=1, \ldots, Q)$ forming a $Q \times Q$ square lattice with periodic boundary conditions. In the literature of solid state theory (for recent textbooks see Refs. [27,28]) the atomic displacements are usually decomposed into two-dimensional Fourier components that are defined by the real and imaginary parts of the following complex functions:

$$
\varphi_{j k}=e^{\frac{2 i \pi}{Q}(j p+k q)},
$$

where $i$ is the imaginary unit, $p$ and $q(p, q=-1,0,+1$ for $Q=3$ ) characterize the horizontal and vertical wave numbers. Accordingly, the Fourier components of the payoffs $A_{j k}$ are given as the real and imaginary parts of $\varphi_{j k}$. The readers can recognize that the previous basis vectors (11) can also be considered as suitable Fourier components for $Q=2$.

For sake of simplicity henceforth our investigation is restricted to the case of $Q=3$ and the corresponding Fourier components $\mathbf{g}(n)$ are labeled by a single number $n=1, \ldots, 9$. The resultant nine components obey the following forms:

$$
\begin{aligned}
& \mathbf{g}(1)=\frac{1}{3}\left(\begin{array}{lll}
1 & 1 & 1 \\
1 & 1 & 1 \\
1 & 1 & 1
\end{array}\right) \\
& \mathbf{g}(2)=\frac{1}{\sqrt{18}}\left(\begin{array}{lll}
-1 & -1 & 2 \\
-1 & -1 & 2 \\
-1 & -1 & 2
\end{array}\right), \\
& \mathbf{g}(3)=\frac{1}{\sqrt{6}}\left(\begin{array}{lll}
1 & -1 & 0 \\
1 & -1 & 0 \\
1 & -1 & 0
\end{array}\right) \\
& \mathbf{g}(4)=\frac{1}{\sqrt{18}}\left(\begin{array}{rrr}
-1 & -1 & -1 \\
-1 & -1 & -1 \\
2 & 2 & 2
\end{array}\right) \text {, } \\
& \mathbf{g}(5)=\frac{1}{\sqrt{6}}\left(\begin{array}{rrr}
1 & 1 & 1 \\
-1 & -1 & -1 \\
0 & 0 & 0
\end{array}\right) \text {, } \\
& \mathbf{g}(6)=\frac{1}{\sqrt{18}}\left(\begin{array}{rrr}
-1 & 2 & -1 \\
2 & -1 & -1 \\
-1 & -1 & 2
\end{array}\right) \text {, } \\
& \mathbf{g}(7)=\frac{1}{\sqrt{6}}\left(\begin{array}{rrr}
-1 & 0 & 1 \\
0 & 1 & -1 \\
1 & -1 & 0
\end{array}\right) \text {, }
\end{aligned}
$$




$$
\begin{aligned}
\mathbf{g}(8) & =\frac{1}{\sqrt{18}}\left(\begin{array}{rrr}
2 & -1 & -1 \\
-1 & 2 & -1 \\
-1 & -1 & 2
\end{array}\right), \\
\mathbf{g}(9) & =\frac{1}{\sqrt{6}}\left(\begin{array}{rrr}
0 & -1 & 1 \\
1 & 0 & -1 \\
-1 & 1 & 0
\end{array}\right),
\end{aligned}
$$

where the prefactors are chosen to satisfy the orthonormal conditions analogous to those given by Eq. (11). Using this set of basis vectors each $3 \times 3$ payoff matrix can be expressed as

$$
\mathbf{A}=\sum_{n=1}^{9} \beta(n) \mathbf{g}(n),
$$

where

$$
\beta(n)=\sum_{j, k=1}^{3} A_{j k} g_{j k}(n) .
$$

Notice that the first component gives a constant contribution to the payoff; therefore, it is an irrelevant term for most of the dynamical rules. The components $\mathbf{g}(2)$ and $\mathbf{g}(3)$ consist of uniform columns, therefore, these terms do not motivate the rational players to choose another strategy, thus these terms give zero contribution to the potential $\mathbf{V}$. The linear combinations of the latter terms together with $\mathbf{g}(1)$ represent the cross-dependent payoffs for the symmetric three-strategy games. Here the self-dependent payoff contributions can be determined by the linear combination of the components $\mathbf{g}(1)$, $\mathbf{g}(4)$, and $\mathbf{g}(5)$. The payoffs are fixed within the rows of these components therefore the corresponding contributions to the potential matrix are equivalent to $\mathbf{g}(4)+\mathbf{g}^{+}(4)$ and $\mathbf{g}(5)+\mathbf{g}^{+}(5)$. Because the components $\mathbf{g}(6), \mathbf{g}(7)$, and $\mathbf{g}(8)$ are symmetric matrices, their contribution to the potentials are defined by themselves. The last component, $\mathbf{g}(9)$, corresponds to the well-known rock-scissors-paper (RSP) game. This is the only component for which the potential does not exist. Thus the symmetric $3 \times 3$ game with payoff matrix $\mathbf{A}$ is a potential game, if and only if $\beta(9)=0$. The absence of the potential for the RSP component is a direct consequence of the fact that, along the closed trajectory $(1,2) \rightarrow(1,3) \rightarrow(2,3) \rightarrow$ $(2,1) \rightarrow(3,1) \rightarrow(3,2) \rightarrow(1,2)$ within the two-player strategy profiles, the payoff variation of the strategy-modifying player is the same $(\sqrt{2 / 3})$ for each step that prohibits the existence of potential [7]. Consequently, potential matrix exist if $\beta(9)=0$ and its value can be given as

$$
\mathbf{V}=\sum_{n=4}^{8} \beta(n) \mathbf{g}(n)+\beta(4) \mathbf{g}^{+}(4)+\beta(5) \mathbf{g}^{+}(5) .
$$

For an illustration of the decomposition now we consider the payoff matrix

$$
\mathbf{A}=\left(\begin{array}{lll}
0 & T & \sigma \\
S & 1 & \sigma \\
\sigma & \sigma & \sigma
\end{array}\right)
$$

which is introduced for the investigation of the voluntary prisoner's dilemma games $(T>1, S<0$, and $0<\sigma<1)$ where the three strategies are the defection, cooperation, and loner declining participation in the game [29-33]. One can easily evaluate the corresponding coefficients $\beta(n)$ defined by Eq. (26). The straightforward calculation gives that all these coefficients differ from zero. Due to the symmetries, however, some of these coefficients [e.g., $\beta(3), \beta(5), \beta(7)$, and $\beta(9)]$ are independent of $\sigma$. Potential does not exist because $\beta(9)=(S-T) / \sqrt{6}$. The quantitative investigations of the corresponding evolutionary game indicated global oscillations in the strategy frequencies for mixed populations $[34,35]$ and self-organizing patterns with rotating spiral arms in the spatial systems [3]. The observed cyclic dominance among the three strategies is interpreted as the Red Queen mechanism [36]. Here it is worth mentioning that, among the nonsymmetric $2 \times 2$ games, the matching pennies component can induce similar effects [37-39].

\section{FEATURES OF THE COMPONENTS}

Due to the absence of real individual motivations among the rational players for the cross-dependent games $[\mathbf{A}=$ $\beta(1) \mathbf{g}(1)+\beta(2) \mathbf{g}(2)+\beta(3) \mathbf{g}(3)]$, all the players choose their strategy at random if the evolution is governed by the logit rule (5). Consequently, in these systems the strategies are selected with equal probability independently of the connectivity structure and noise level $K$.

The direct pair interactions are also missing for the self-dependent components $\left[\mathbf{A}^{\text {(self) }}=\beta(1) \mathbf{g}(1)+\beta(4) \mathbf{g}(4)+\right.$ $\beta(5) \mathbf{g}(5)]$ that can be given as $A_{j k}^{\text {(self) }}=\mu_{j}=\frac{1}{3} \sum_{k} A_{j k}$ (for $j, k=1,2,3)$. Here $\mu_{j}$ is the average payoff of the first player if she chooses strategy $j$ whereas her co-player uses a mixed strategy with selecting the three options with equal probability. Within the game theory $\mu_{j}$ quantifies the driving force of risk dominance introduced by Harsanyi and Selten [40]. At the same time, in the terminology of three-state Potts $\mu_{j}$ represents the external field favoring or suppressing the presence of the $j$ th state. If $\mathbf{A}=\mathbf{A}^{\text {(self) }}$ then each player chooses the $j$ th strategy with a frequency

$$
\rho_{j}=\frac{e^{z \mu_{j} / K}}{\sum_{l} e^{z \mu_{j} / K}},
$$

where $z$ denotes the number of neighbors for regular connectivity structures. Evidently, all the players choose their best choice in the limit $K \rightarrow 0$.

In the literature of physics the models with the last two types of games are also well investigated because $\mathbf{g}(8)$ represents the three-strategy coordination game or the Potts model [19] that are already investigated in the presence of external magnetic fields [ $\mathbf{g}(4)$ and $\mathbf{g}(5)]$ or a RSP component $[\mathbf{g}(9)][3,41]$. The three-state Potts model describes an order disorder transition if the noise level $K$ is increased. At low noise the system evolves into a state dominated by one of the three strategies with some isolated point defects. The increase of $K$ results in more frequent and larger defects inside the ordered structure and above a threshold value $\left(K>K_{c}\right)$ all the three strategies are present with the same frequency in the stationary state. The resultant transition is usually quantified by evaluating frequencies $\rho_{j}(j=1,2,3)$ as a function of $K$ as it is illustrated in Fig. 1.

The plotted critical transition represents a universal behavior in systems that belong to a universality class named after the three-state Potts model. For one of the possible ordered 


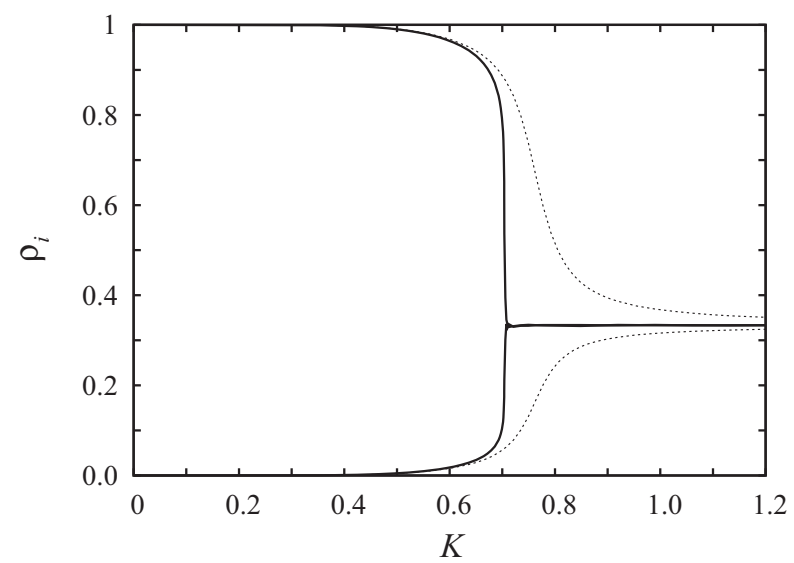

FIG. 1. Average frequencies of strategies are illustrated by solid lines as a function $K$ for the Potts model in the absence of external field $[\mathbf{A}=\mathbf{g}(8)]$. Dashed lines show the same quantities if $\mathbf{A}=\mathbf{g}(8)+$ $\varepsilon \mathbf{g}(4)$ for $\varepsilon=0.01$.

states the $K$ dependence of the strategy frequencies can be given by a single parameter as

$$
\begin{aligned}
& \rho_{1}(K)=\rho_{2}(K)=\frac{1}{3}-\gamma(K), \\
& \rho_{3}(K)=\frac{1}{3}+2 \gamma(K),
\end{aligned}
$$

where $\gamma(K)=0$ if $K>K_{c}, \gamma(K)=1 / 3$ if $K \rightarrow 0$, and it vanishes algebraically $\left[\gamma(K) \propto\left(K_{c}-K\right)^{1 / 9}\right]$ if $K$ approaches $K_{c}$ from below. The other two possible states at low noise can be obtained by cyclic permutation of the strategy labels.

The general and universal features of this transition and also the accompanying phenomena are well described in a review of Potts model [17] and textbooks [42-44] of statistical physics. Now we illustrate only that this critical transition is smoothed out for the application of a suitable external field as these results serve as references for the discussion of other models. The plotted strategy frequencies $\rho_{i}$ in Fig. 1 are obtained by Monte Carlo simulations for sufficiently large sizes and long sampling times providing small statistical errors that are comparable to the line thickness in Fig. 1.

Figure 1 compares typical variations in the strategy frequencies versus $K$ if $\mathbf{A}=\mathbf{g}(8)+\beta(4) \mathbf{g}(4)[\beta(4) \geqslant 0]$. Evidently the plotted curves tend towards the results of Potts model if $\beta(4) \rightarrow+0$. In the latter case the third strategy is preferred by the positive term of $\mathbf{g}(4)$ whereas the others are suppressed equally. With a suitable choice of the coefficients $\beta(1), \beta(4)$, and $\beta(5)$ one can tune these preferences separately for each player.

In the absence of the external field(s) or self-dependent components $[\mathbf{g}(4)=\mathbf{g}(5)=0]$ a domain growing process can be observed at low noise level if the system is started from a random initial state. In that case the interfaces separating the three domains move randomly with a zero average invasion velocity. In the presence of external fields the preferred domains invade the territories of others with an average velocity proportional to the difference in strength of the driving fields. Due the latter phenomena the system develops into a mono-domain state of the preferred strategy.

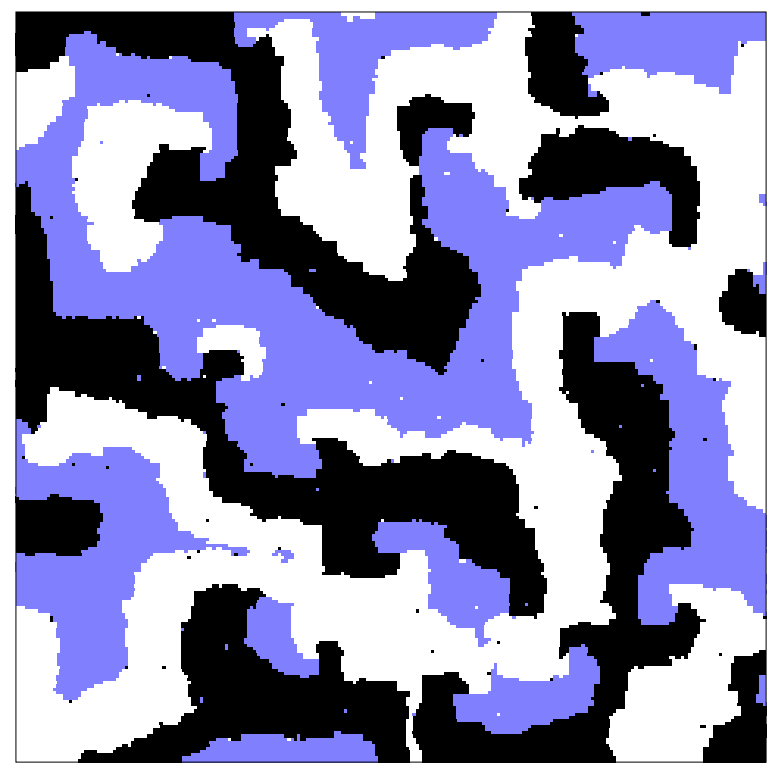

FIG. 2. (Color online) Typical snapshot of self-organizing strategy distributions on a square lattice for a three-strategy model where the pair interaction is described by $\mathbf{A}=\mathbf{g}(8)+\beta(9) \mathbf{g}(9)$ for $\beta(9)=0.1$ at a low noise level $(K=0.3)$.

Basically different behaviors can be observed when the rock-scissors-paper $[\mathbf{g}(9)]$ component disturbs the domain growing process. Namely, for $\beta(8)=1$ and $|\beta(9)| \ll 1$ the three homogeneous domains invade cyclically each other with an average velocity proportional to $\beta(9)$ at low noise. These cyclical invasions block the domain growing process and finally the system evolves into a self-organizing pattern with rotating spirals around the vertices where three types of interfaces meet, as it is illustrated in Fig. 2. More precisely, one can distinguish two types of rotating vertices, called vortices and antivortices, that rotate clockwise and anticlockwise for $\beta(9)>0$. Evidently, the direction of rotation is reversed simultaneously for both types of vortices if the sign of $\beta(9)$ is reversed. Similar rotating spirals are observed in many three-strategy models for different dynamical rules (for a brief survey see Ref. [3] with further references therein).

The above decomposition implies that the appearance of this type of self-organizing pattern is strongly related to the presence of the RSP component $[\beta(9) \neq 0]$. Previous investigations have quantified that the correlation length and the average distance between the vertices and antivertices diverge if the driving force vanishes $[\beta(9) \rightarrow 0]$ independently of the presence and absence of the (attractive) Potts-type interactions [45-47].

The rest of the nine components are discussed separately in the following sections because the resultant states require a more complex characterization.

\section{COORDINATIONS}

First we briefly study the component $\mathbf{g}(6)$, which can be transformed into $\mathbf{g}(8)$ by exchanging the first and second rows of the matrix. Similar transformation is used to justify the equivalence between the behavior of the ferromagnetic 


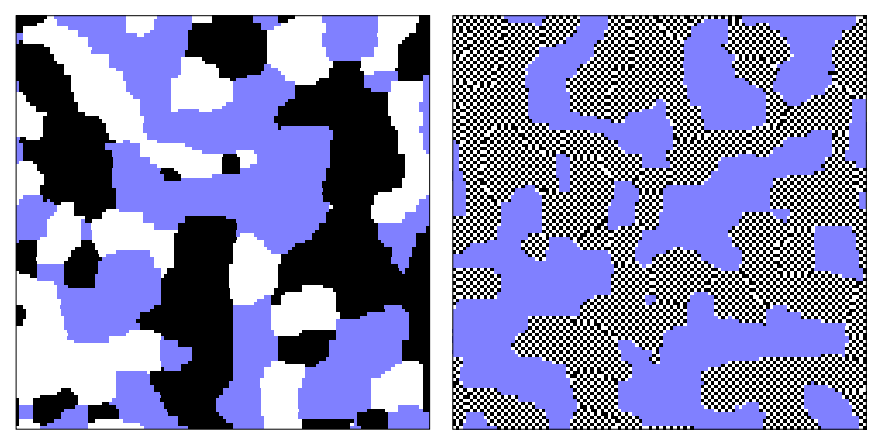

FIG. 3. (Color online) Snapshots illustrate typical strategy distributions during the domain growing process on a square lattice at a low noise level if the interaction is described by $\mathbf{g}(8)$ (left) and $\mathbf{g}(6)$ (right). For both cases the finite system evolves into a mono-domain state dominated by one of the three ordered strategy arrangements.

and antiferromagnetic Ising models in the absence of an external magnetic field. The quantification of the mentioned equivalence requires the division of the lattice into two identical sublattices (denoted $v=1$ and 2) that in the present square lattice can be done on the analogy of the black and white boxes of the chessboard. In that case the stationary states of these systems can be characterized by $\rho_{j}^{(\nu)}(K)$ denoting the average frequency of the $j$ th strategy as a function of $K$ in the sublattice $v$.

The peculiarity of the possible states of the system for $\mathbf{A}=\mathbf{g}(6)$ at low noise levels is illustrated in Fig. 3 where a typical strategy distribution is shown in the course of domain growing at time $t=5000 \mathrm{MCS}$. In that case the systems are started from a random initial state for both types of interactions. The right-hand snapshot illustrates the formation of three domains. The homogeneous domain is formed by strategy 3 whereas the second and third ones correspond to chessboard and antichessboard arrangements of the strategies 1 and 2 . The interfaces separating the three domains move in the same way as it observed for the three-state Potts model. For the sake of comparison the left-hand snapshot shows the strategy distribution for the three-state Potts model $\mathbf{A}=\mathbf{g}(8)$ at the same time, size, and noise level. The reader can easily recognize that the right-hand pattern becomes similar to the left-hand one if the black and white colors are exchanged within one of the sublattices. For any finite sizes after a sufficiently long transient time the system evolves into a state where one of the three ordered strategy arrangements prevails the whole system as it happens for the Potts model, too.

If the homogeneous phase is realized for $\mathbf{A}=\mathbf{g}(6)$ then the strategy frequencies are equivalent in both sublattices for $K<K_{c}$; that is,

$$
\begin{aligned}
& \rho_{1}^{(\nu)}(K)=\rho_{2}^{(\nu)}(K)=\frac{1}{3}-\gamma(K), \\
& \rho_{3}^{(\nu)}(K)=\frac{1}{3}+2 \gamma(K),
\end{aligned}
$$

where $v=1,2$ and the value of $\gamma(K)$ is the same obtained for the Potts model [see Ref. (30)]. Besides it, this system has two additional (equivalent) solutions at low noise levels; namely,

$$
\begin{aligned}
& \rho_{1}^{(2)}(K)=\rho_{2}^{(1)}(K)=\frac{1}{3}-\gamma(K), \\
& \rho_{3}^{(1)}(K)=\rho_{3}^{(2)}(K)=\frac{1}{3}-\gamma(K), \\
& \rho_{1}^{(1)}(K)=\rho_{2}^{(2)}(K)=\frac{1}{3}+2 \gamma(K),
\end{aligned}
$$

and the third solution can be given by exchanging the sublattice indices in Eqs. (32). Equation (31) is the solution that contains only strategy 3 for $K \rightarrow 0$, while Eq. (32) and its counterpart converge when $K \rightarrow 0$ to chessboard and antichessboard arrangements of strategies 1 and 2 with no presence of strategy 3.

One can easily recognize that there exist two further systems possessing similar set of solutions. The common features of these systems are the existence of three equivalent solutions at the low-noise limit. One of these solutions is the homogeneous strategy distribution whereas there exist two equivalent chessboard- and antichessboard-like arrangements formed by the second and third strategies as illustrated in the right-hand snapshot of Fig. 3. Straightforward calculations give that the corresponding two games can be expressed as

$$
-\frac{1}{2} \mathbf{g}(6)+\frac{\sqrt{3}}{2} \mathbf{g}(7)=\frac{1}{\sqrt{18}}\left(\begin{array}{rrr}
2 & -1 & -1 \\
-1 & -1 & 2 \\
-1 & 2 & -1
\end{array}\right)
$$

and

$$
-\frac{1}{2} \mathbf{g}(6)-\frac{\sqrt{3}}{2} \mathbf{g}(7)=\frac{1}{\sqrt{18}}\left(\begin{array}{rrr}
-1 & -1 & 2 \\
-1 & 2 & -1 \\
2 & -1 & -1
\end{array}\right) .
$$

Accordingly, the family of the three-state Potts models is represented by four payoff matrices defined by $\mathbf{g}(8), \mathbf{g}(6)$, and the expressions (33) and (34). These payoff matrices have some common features. Namely, all of these matrices are symmetric and the elements of matrices are defined by one of the two possible values. In general the maximum value of the potential matrix is achieved for the Nash equilibrium. Here there are three equivalent maximum values (one for each column and row) and the corresponding $j$ and $k$ indices of the maxima can be considered as three strict Nash equilibria for the given two-player game. In the light of the above results the vector space spanned by $\mathbf{g}(6)$ and $\mathbf{g}(7)$ contains blends of coordination and anticoordination. Within this space, there are three equivalent games-represented by $\mathbf{g}(6)$ and Eqs. (33) and (34) - in which one strategy prefers to coordinate itself and the other two prefer to anticoordinate with each other.

In fact, these four models represent similar types of possible situations. Within the terminology of game theory all these systems can be interpreted as coordination games if the players use suitable strategy labels.

Three of these models are located within a two-dimensional subspace spanned by $\mathbf{g}(6)$ and $\mathbf{g}(7)$ and denoted by closed circles in Fig. 4. As this subspace has some other curiosity therefore now we study the models

$$
\mathbf{A}=\cos (\varphi) \mathbf{g}(6)+\sin (\varphi) \mathbf{g}(7),
$$




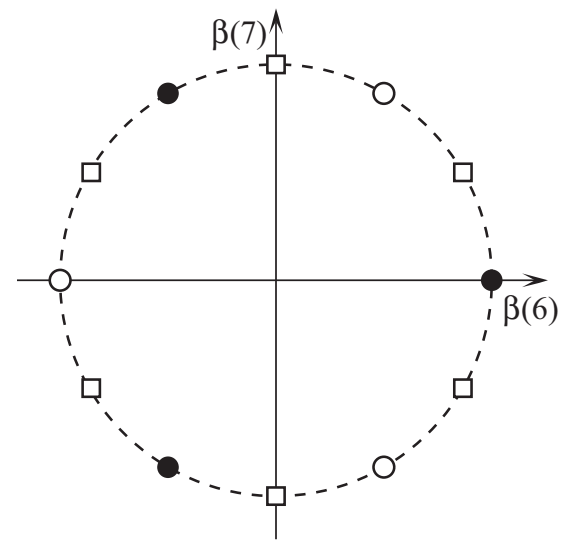

FIG. 4. Games defined by Eq. (35) are located along the dashed line in the two-dimensional subspace of parameters. Closed circles represent games related closely to the attractive Potts model. Open circles illustrate models equivalent to the repulsive three-state Potts model. Open squares show six models that can be transformed into $\mathbf{g}(6)$ by the permutation of the strategy labels.

which are identified by the value of $\varphi$. For $\varphi=0$ or $\pi / 2$ the game is equivalent to $\mathbf{g}(6)$ or $\mathbf{g}(7)$. These games have a common feature. Namely, all of these games have three strict Nash equilibria with equivalent payoffs and potential values (excepting the models indicated by open circles in Fig. 4) as the model given by the payoff matrix (34). Consequently, similar behavior is expected at low noise levels as demonstrated by the $K$ dependence of the strategy frequencies within the sublattices. Contrary to the above-mentioned members of the Potts model family, here quantities $\rho_{j}^{(v)}(K)$ are characterized by two distinct parameters: $\gamma_{1}(K)$ and $\gamma_{2}(K)$. For example, one of the possible three equivalent solutions for $\mathbf{A}=\mathbf{g}(7)$ can be given as

$$
\begin{aligned}
& \rho_{1}^{(1)}(K)=\rho_{1}^{(2)}(K)=\frac{1}{3}-\gamma_{1}(K), \\
& \rho_{2}^{(1)}(K)=\rho_{2}^{(2)}(K)=\frac{1}{3}+\gamma_{1}(K)+\gamma_{2}(K), \\
& \rho_{3}^{(1)}(K)=\rho_{3}^{(2)}(K)=\frac{1}{3}-\gamma_{2}(K),
\end{aligned}
$$

where $\gamma_{1}(K)=\gamma_{2}(K)=0$ if $K>K_{c}, \gamma_{1}(K)=\gamma_{2}(K)=1 / 3$ if $K \rightarrow 0$ in analogy with the Potts model where these two parameters coincide and the value of $K_{c}$ depends on $\varphi$. The latter expressions define the state when only strategy 2 takes place on the square lattice in the limit $K \rightarrow 0$ whereas the minor strategies are present with different frequencies at low noise, as illustrated in Fig. 5.

In this model, however, there are two equivalent states with chessboard-like arrangements of the strategies 1 and 3. For one of these solutions the strategy frequencies in the sublattices can be expressed by the same two parameters as

$$
\begin{aligned}
& \rho_{1}^{(1)}(K)=\rho_{3}^{(2)}(K)=\frac{1}{3}+\gamma_{1}(K)+\gamma_{2}(K), \\
& \rho_{2}^{(1)}(K)=\rho_{2}^{(2)}(K)=\frac{1}{3}-\gamma_{2}(K), \\
& \rho_{3}^{(1)}(K)=\rho_{1}^{(2)}(K)=\frac{1}{3}-\gamma_{1}(K),
\end{aligned}
$$

and the third solution can be obtained by exchanging the sublattice indices.

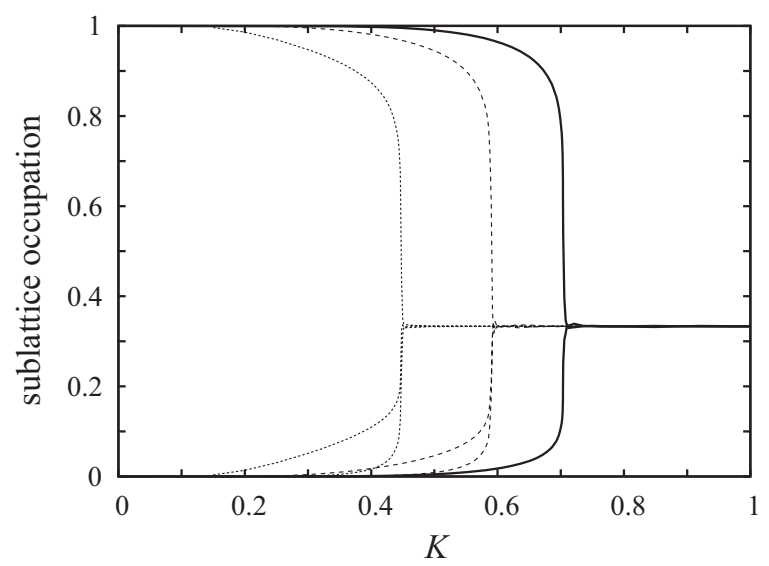

FIG. 5. Average strategy frequencies in the sublattices as a function of $K$ for the interactions defined by Eq. (35). Solid, dashed, and dotted lines show the MC results for $\varphi=0, \pi / 6$, and $\pi / 4$, respectively.

The numerical results in Fig. 5 are obtained from simulations started from one of the ordered states at a low noise level. After a suitable thermalization time, the average frequencies are determined over a sampling time. Subsequently, the noise level is increased and both the thermalization and sampling are repeated as before. MC data obtained when starting the simulations from a different ordered state confirm the results summarized in Eqs. (36) and (37).

There are several basic features that make this system similar to those defined by $\mathbf{g}(6)$ or Eqs. (33) or (34). Namely, the common features are the similar symmetries and the presence of three maximum values (strict Nash equilibria) that are located along the antidiagonal [as well as for Eq. (34)]. On the other hand, the payoff matrix (or potential) $\mathbf{g}(7)$ has three different elements $(-1,0$, and +1$)$ that are located parallel with the antidiagonal. As a result, one can create five additional equivalent systems with a payoff matrix obtained from $\mathbf{g}(7)$ by permutating the rows and columns simultaneously. These equivalent games are located within the plane spanned by $\mathbf{g ( 6 )}$ and $\mathbf{g ( 7 )}$ and are illustrated by six open boxes in Fig. 4. Interestingly, this subset is closed under multiplying the payoff matrices by -1 . The striking consequence of this additional symmetry is illustrated in Fig. 6. At first glance

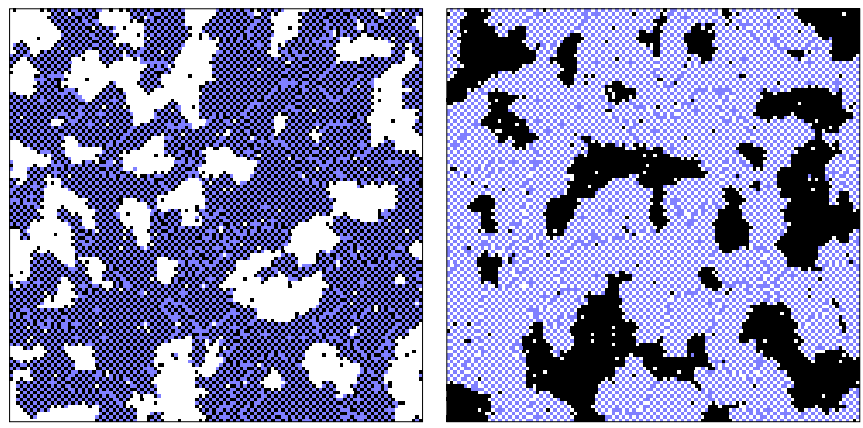

FIG. 6. (Color online) Typical snapshots illustrating strategy distributions during the domain growing process at a low noise level if the interactions are given by (left) $\mathbf{g}(7)$ and (right) $-\mathbf{g}(7)$. 


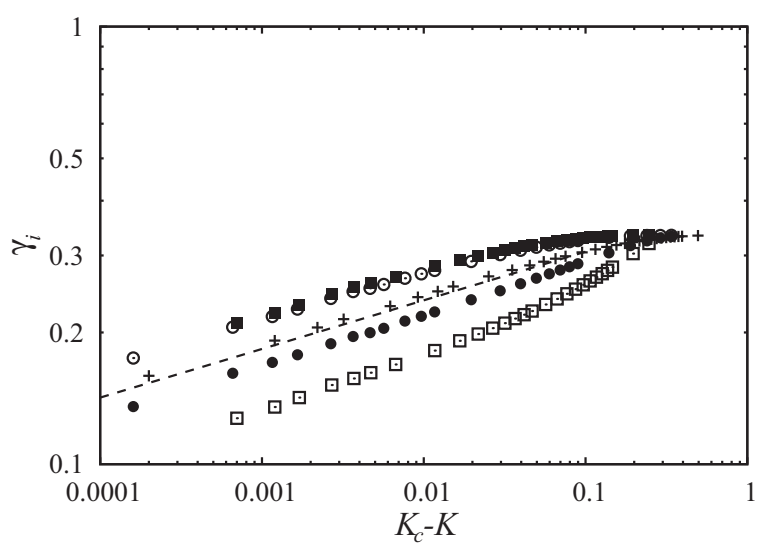

FIG. 7. Log-log plot of $\gamma_{1}$ and $\gamma_{2}$ versus $K_{c}-K$ for $\varphi=0$ [crosses, here $\gamma_{1}=\gamma_{2}$, and $K_{c}=0.9952$ (1)], $\pi / 6$ [open and closed circles, for $K_{c}=0.58966$ (2)], and $\pi / 4$ [open and closed boxes, for $K_{c}=0.4467$ (1)]. The dashed line refers to the theoretical prediction (with a slope of 1/9) for the Potts model.

these snapshots are similar to those plotted on the right side of Fig. 3 as one can distinguish one homogeneous and two chessboard-like strategy arrangements within the three types of growing domains. It can be recognized, however, that all of these ordered structures are decorated by two types of point defects appearing with different frequencies quantified by the parameters $\gamma_{1}(K)$ and $\gamma_{2}(K)$ in the expressions (31) and (32). According to our preliminary results both of these quantities decrease algebraically if $K$ goes to a critical value $K_{c}$.

Figure 7 illustrates the power-law behavior of $\gamma_{1}(K)$ and $\gamma_{2}(K)$ when approaching the critical noise level $K_{c}$ for three values of $\varphi$ characterizing the models within the this two-dimensional subspace. The plotted $\mathrm{MC}$ results are consistent with the expectations predicting $\gamma_{1}(K), \gamma_{2}(K) \propto$ $\left(K_{c}-K\right)^{1 / 9}$ if $\left|K_{c}-K\right| \ll K_{c}$. This behavior is characteristic of the universality class named after the three-state Potts model where the ordering process is described by only a one-dimensional order parameter $\left[\gamma_{1}(K)=\gamma_{2}(K)\right][19,48]$.

Finally we briefly discuss the systems that are equivalent to $-\mathbf{g}(6)$ and denoted by open circles in Fig. 4. In agreement with the previous discussions these interactions are related to the repulsive three-state Potts model that is not capable to form long-range ordered strategy arrangements on a square lattice at low noise $[19,49]$. The absence of ordered strategy arrangements is related to the frustration studied well in the literature of statistical physics [50]. Here the frustration is indicated by the absence of strict Nash equilibria. More precisely, the present payoff (or potential) matrices have six equivalent maxima but none of them are strict Nash equilibria. For the corresponding strategy profiles, however, one of the players can deviate without reducing its own payoff, leading to the appearance of frustration.

\section{SUMMARY}

For the multi-agent spatial evolutionary games the symmetric pair interactions are generally defined by $Q \times Q$ payoff matrices that can be considered as $Q^{2}$-dimensional vectors. It turns out that these interactions can be constructed as a sum of orthonormal basis vectors (with suitable coefficients) that are represented by the two-dimensional Fourier components of the payoff matrix. The systematic analysis of these Fourier components for $Q=2$ and 3 has allowed us to identify relevant and irrelevant terms within the pair interactions. For example, $Q$ of $Q^{2}$ terms (called cross-dependent components) do not influence the players' decision if they wish improve their own payoffs independently of others. If the payoffs or interactions involve only these terms then the players choose their strategy at random for the sequential myopic strategy updates, which happens for the logit rule. Furthermore, there are $Q$ terms for which the payoff depends only on one's own strategy. For games containing only these terms, all players independently reach their own optimum payoff in the low-noise limit.

Real pair interactions are realized for the coordination-type components that favor the choice of the same or different strategies depending on the sign of the corresponding coefficients. Within this subset of games the exchange of strategy labels for one of the players will not modify the essence of coordination. In the case of two strategies, such coordination and anticoordination games are equivalent to the Ising model with ferromagnetic and antiferromagnetic interactions [17]. For the three-strategy games this type of equivalence is extended due to the larger number of possible exchanges in the strategy labels. As a result, we have found three additional games or interactions that yield behavior similar to that described by the three-state Potts model that realizes one of the three equivalent ordered strategy arrangements at low-noise levels. Interestingly, for a suitable combination of the latter games the spatial evolutionary games (with logit dynamical rule) exhibit an order-disorder phase transition when varying the noise level. Due to the difference in the frequency of the minor strategies the phase transition can be quantified by a two-dimensional order parameter that vanishes algebraically when approaching the critical transition point(s). The latter systems can extend the family of models belonging to universality class of the three-state Potts model.

It is found that one of the nine Fourier components is identical to the rock-scissors-paper (RSP) game and in the absence of the RSP component the game is a potential game. Thus the evaluation of the corresponding coefficient $[\beta(9)]$ gives us a simple way to determine whether a symmetric 3 game is a potential game or not.

The Fourier decomposition represents a matrix in a way that highlights certain symmetries that can be exploited in the analysis of spatial evolutionary games. This decomposition may also be useful for other types of interactions characterized by a matrix, e.g., population dynamics of ecological systems [41,51]. On the other hand, there may be other matrix decompositions that better reflect the relevant symmetries of the systems.

\section{ACKNOWLEDGMENTS}

This work was supported by the John Templeton Foundation (FQEB Grant \#RFP-12-22), the Hungarian National ResearchFund (OTKA TK-101490), and the European Social Fund through project FutureICT.hu (TAMOP-4.2.2.C11/1/KONV-2012-0013). 
[1] J. von Neumann and O. Morgenstern, Theory of Games and Economic Behaviour (Princeton University Press, Princeton, 1944).

[2] M. A. Nowak, Evolutionary Dynamics (Harvard University Press, Cambridge, 2006).

[3] G. Szabó and G. Fáth, Phys. Rep. 446, 97 (2007).

[4] B. Allen and M. A. Nowak, EMS Surv. Math. Sci. 1, 113 (2014).

[5] M. A. Nowak and R. M. May, Nature (London) 359, 826 (1992).

[6] E. Lieberman, C. Hauert, and M. A. Nowak, Nature (London) 433, 312 (2005).

[7] D. Monderer and L. S. Shapley, Games Econ. Behav. 14, 124 (1996).

[8] D. Monderer and L. S. Shapley, J. Econ. Theory 68, 258 (1996).

[9] L. E. Blume, Games Econ. Behav. 5, 387 (1993).

[10] L. E. Blume, Games Econ. Behav. 11, 111 (1995).

[11] A. V. M. Herz, J. Theor. Biol. 169, 65 (1994).

[12] E. Callen and D. Shapero, Phys. Today 27, 23 (1974).

[13] H. Föllmer, J. Math. Econ. 1, 51 (1974).

[14] W. Weidlich, Phys. Rep. 204, 1 (1991).

[15] R. J. Glauber, J. Math. Phys. 4, 294 (1963).

[16] E. Ising, Z. Phys. 31, 253 (1925).

[17] C. Domb, in Phase Transitions and Critical Phenomena, edited by C. Domb and M. S. Green (Academic Press, London, 1974), Vol. 3, pp. 357-484.

[18] R. B. Potts, Math. Proc. Cambridge Philos. Soc. 48, 106 (1952).

[19] F. Y. Wu, Rev. Mod. Phys. 54, 235 (1982).

[20] S. Galam and B. Walliser, Phys. A (Amsterdam, Neth.) 389, 481 (2010).

[21] D. Fudenberg and D. Levine, The Theory of Learning in Games (MIT Press, Cambridge, 1998).

[22] J. Hofbauer and W. H. Sandholm, J. Econ. Theor. 132, 47 (2007).

[23] W. H. Sandholm, Population Games and Evolutionary Dynamics (MIT Press, Cambridge, 2010).

[24] L. Boltzmann, Sitzunber. Kais. Akad. Wiss. Wien Math. Naturwiss. Classe 76, 373 (1877).

[25] T. M. Liggett, Interacting Particle Systems (Springer, New York, 1985).

[26] B. Wu, B. Bauer, T. Galla, and A. Traulsen, arXiv:1406.4030.

[27] C. Kittel, Introduction to Solid State Physics (John Wiley \& Sons, Chichester, 2004).
[28] J. Sólyom, Fundamentals of the Physics of Solids, Volume 1, Structure and Dynamics (Springer-Verlag, Berlin, 2007).

[29] G. Szabó and C. Hauert, Phys. Rev. E 66, 062903 (2002).

[30] C. Hauert and G. Szabó, Complexity 8, 31 (2003).

[31] G. Szabó and J. Vukov, Phys. Rev. E 69, 036107 (2004).

[32] Z.-X. Wu, X.-J. Xu, Y. Chen, and Y.-H. Wang, Phys. Rev. E 71, 037103 (2005).

[33] Y. Chen, S.-M. Qin, L. Yu, and S. Zhang, Phys. Rev. E 77, 032103 (2008).

[34] C. Hauert, S. De Monte, J. Hofbauer, and K. Sigmund, Science 296, 1129 (2002).

[35] K. Sigmund, The Calculus of Selfishness (Princeton University Press, Princeton, 2010).

[36] L. van Valen, Evol. Theory 1, 1 (1973).

[37] L. van Valen, Evol. Theory 4, 129 (1980).

[38] G. Szabó, L. Varga, and I. Borsos, Phys. Rev. E 89, 042820 (2014).

[39] Z. Cao and X. Yang, Theor. Comput. Sci. 540-541, 169 (2014).

[40] J. C. Harsanyi and R. Selten, A General Theory of Equilibrium Selection in Games (MIT Press, Cambridge, 1988).

[41] J. Hofbauer and K. Sigmund, Evolutionary Games and Population Dynamics (Cambridge University Press, Cambridge, 1998).

[42] D. Chandler, Introduction to Modern Statistical Mechanics (Oxford University Press, Oxford, 1987).

[43] K. Toda, R. Kubo, and N. Saito, Statistical Physics I: Equilibrium Statistical Mechanics (Springer, Berlin, Heidelberg, 1991).

[44] D. A. Lavis and G. M. Bell, Statistical Mechanics of Lattice Systems: I (Springer, Berlin, 1999).

[45] K. Tainaka and Y. Itoh, Europhys. Lett. 15, 399 (1991).

[46] G. Szabó and A. Szolnoki, Phys. Rev. E 65, 036115 (2002).

[47] J. Vukov, A. Szolnoki, and G. Szabó, Phys. Rev. E 88, 022123 (2013).

[48] H. E. Stanley, Introduction to Phase Transitions and Critical Phenomena (Clarendon Press, Oxford, 1971).

[49] A. N. Berker and L. P. Kadanoff, J. Phys. A: Math. Gen. 13, L259 (1980).

[50] H. T. Diep, Frustrated Spin Systems (World Scientific, Singapore, 2004).

[51] J. Maynard Smith, Evolution and the Theory of Games (Cambridge University Press, Cambridge, 1982). 